Wissen auch Sie manchmal nicht, an wen Sie sich mit einem kniffligen fachlichen oder organisatorischen Problem wenden können? Wir laden Sie ein,

uns Ihre Frage zu schreiben oder zu mailen (DHZ-Redaktion@thieme.de). Bitte haben Sie Verständnis dafür, dass in dieser Rubrik ausschließlich allgemeine therapeutische Fragestellungen diskutiert werden. Fragen zu konkreten Fällen können wir leider nicht beantworten.

\title{
Wie wirkt sich die pauschale Beihilfe auf die Erstattung von Heilpraktilkerleistungen aus?
}

Frage: Ich bin Heilpraktikerin in Hamburg und behandle auch einige verbeamtete Lehrer. Nun habe ich erfahren, dass Beamte in Hamburg seit Anfang August statt einer individuellen Beihilfe eine pauschale Beihilfe beantragen können. Wie funktioniert das? Hat das Auswirkungen auf die Erstattung meiner Leistungen als Heilpraktikerin durch die Beihilfe?

\section{Anonym}

\section{Antwort:}

Sehr geehrte Kollegin,

pauschale Beihilfe bedeutet, dass Beamte in Hamburg und ausschließlich dort - einen 50-Prozent-Zuschuss erhalten, wenn sie sich gesetzlich krankenversichern. Der Beihilfe-Betrag liegt etwa in Höhe der üblichen Arbeitgeberbeiträge für die Krankenversicherung.

Die schlechte Nachricht für uns Heilpraktiker: Bei der pauschalen Beihilfe hat der Beamte keinen Anspruch mehr auf die Erstattung von Heilpraktikerleistungen. Für Sie als Heilpraktikerin bedeutet das, dass Ihre Behandlungen nur dann weiterhin teilweise oder ganz erstattet werden, wenn Ihre verbeamteten Patienten eine Zusatzversicherung wählen, die Heilpraktikerleistungen inkludiert. Ansonsten müssten sie Ihre Leistungen komplett aus der eigenen Tasche honorieren. Sollte einer Ihrer verbeamteten Patienten Sie also nach Ihrer Meinung zur pauschalen Beihilfe fragen, wäre bezogen auf die Erstattung von Heilpraktikerleistungen ganz klar davon abzuraten.

Ich habe bei der Verbraucherzentrale Hamburg e.V. angerufen, um eine Einschätzung über die pauschale Beihilfe zu erhalten. Man scheint - das verwundert mich - dort viel von diesem neuen Modell zu halten. Die Argumentation: Vor allem ältere Patienten würden davon profitieren, da sie keine Rechnungen mehr an die Beihilfe und die Privatversicherung senden müssen und dadurch weniger Aufwände hätten. Ich habe jedoch die Erfahrung gemacht, dass dies auch für ältere Patienten kein Problem ist, da sie an diese Abläufe gewöhnt sind, sie fast wie im Schlaf beherrschen. Und die Vorteile als Privatpatient halte ich gerade im Alter, wenn die schlimmen Krankheiten drohen, für einen nicht zu unterschätzenden Vorteil. Doch hat der Beamte die pauschale Beihilfe einmal gewählt, gibt es kein Zurück mehr. Die Entscheidung ist unwiderruflich. Das ist ein ganz klarer Nachteil.

HP Siegfried Kämper, Gelsenkirchen

Dieser Artikel ist online zu finden:

http://dx.doi.org/10.1055/a-0638-5506

\section{Gelenkbeschwerden?}

\author{
Homöopathische Regeneration \\ der Zellfunktionen mit der \\ Biomolekularen vitOrgan-Therapie
}

\section{www.vitorgan.de}

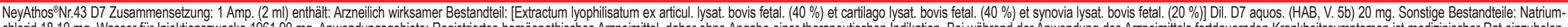

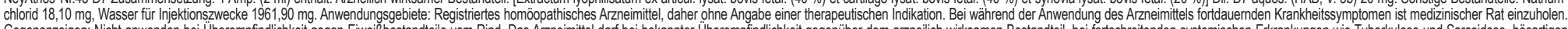

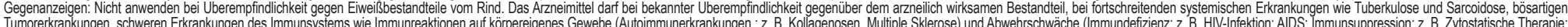

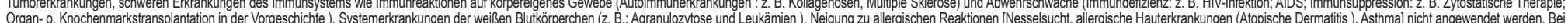

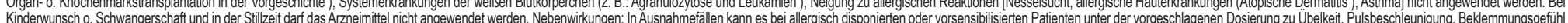

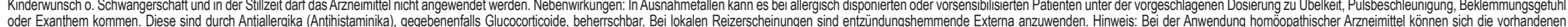

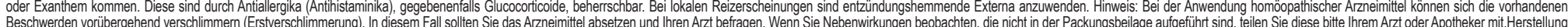

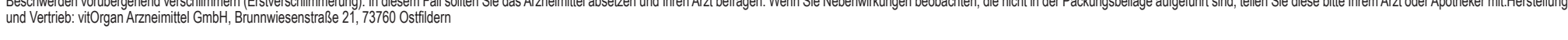

